\title{
Le rôle de l'église et de la morale chrétienne sur le contrôle des naissances dès l'Antiquité à nos jours
}

\author{
Georges ANDROUTSOS, Christos ZOURNAS, Aristide DIAMANTIS
}

Histoire de la Médecine, Faculté de Médecine, Université d'Athènes, Grèce

\section{RESUME}

Cet article traite du rôle de l'église, dès ses débuts à nos jours, sur le contrôle des naissances.

Mots clès : église, morale chrétienne, contrôle des naissances

\section{INTRODUCTION}

Avant le $20^{\mathrm{e}}$ siècle et depuis le début du christianisme, le problème qui se posait à l'église était de concilier mariage et chasteté et justifier le mariage tout en maintenant la prééminence de l'état virginal. Pour comprendre donc l'attitude de l'église envers le problème du contrôle des naissances, il faut étudier l'ensemble de sa doctrine du mariage, et chercher d'où viennent les impératifs contradictoires de virginité et de procréation.

\section{LE NOUVEAU TESTAMENT}

Le Nouveau Testament a glorifié la chasteté et la virginité. Les quatre évangélistes et saint Paul témoignent de cette nécessité de la chasteté qui est giorifiée par le célibat exemplaire du Christ ainsi que par la virginité triomphante de Marie. Et le mariage est fini ? Certains disciples hétérodoxes le soutiendront bientôt et, dès les temps apostoliques, beaucoup de chrétiens s'interrogent. La prééminence de l'état virginal, affirmé avec continuité des origines à nos jours, y conduisait naturellement.

Tout en affirmant cette prééminence, saint Paul permet cependant le mariage monogamique. Cette permission est donc donnée pour éviter la fornication, c'est-à-dire pour assurer une forme mineure de la chasteté à ceux et celles qui ne sont pas doués à la continence sexuelle. Curieusement, c'est la chasteté qui justifie le mariage. Le mariage est pourtant plus qu'un remède puisque le Christ en a rappelé l'origine divine et son indissolubilité [3].

Symbole d'amour, remède contre la fornication, indissolubilité, devoir réciproque des époux : voilà comment se définit le mariage dans le Nouveau Testament. Nulle part on n'y insiste sur le bien de procréation, personne n'en parlera avant la fin du ler siècle. Si les droits conjugaux sont limités, c'est essentiellement dans le temps : "Ne vous refusez pas l'un à l'autre, écrit saint Paul aux Corinthiens, à moins que ce ne soit d'un commun accord, pour un temps, afin de vaquer à la prière». II s'agit apparemment d'une continence rituelle, conforme à la tradition hébraïque ; d'une transition nécessaire entre les œuvres profanes et les cuvres sacrées ; ou peut-être déjà d'une précaution contre des tendances hérétiques qui apparaîtront nettement par la suite. Mais aucune limitation explicite quant aux formes et aux fins de l'accouplement.

En Romains I, 26 - 27, on trouve le concept de «rapport contre nature». Saint Paul s'écrie : «leurs femmes ont échangé les rapports naturels pour des rapports contre nature ; pareillement les hommes délaissant l'usage naturel de la femme ont brûlé de passion les uns pour les autres... ". Mais c'est aux relations homosexuelles, déjà condamnées par l'Ancien Testament, que Paul applique ce concept, non aux rapports conjugaux intrinsèquement stériles. Finalement rien, dans les parties les plus anciennes et les plus incontestables du Nouveau Testament, ne fait de la procréation la fin principale du mariage ; rien ne consacre le caractère éminemment respectable qu'elle avait dans l'Ancien, et rien, malgré la référence de Paul à la nature, ne paraît condamner la contraception. C'est après le $\mathrm{I}^{\mathrm{er}}$ siècle,

\section{Correspondance :}

Professeur Georges ANDROUTSOS, 1 rue Ipeirou, 10433, Athènes, Grèce - Email Iyon 48@otenet. gr 
sous l'infiuence d'idées étrangères à la tradition judéo-chrétienne, que l'orthodoxie, se précisant face aux hérésies, va fonder le mariage sur la procréation.

\section{LA CULTURE GRÉCO-ROMAINE}

Parmi ces idées, celles des stoïciens paraissent avoir rencontré une audience particulière dans l'Eglise. Ils visaient à libérer I'homme de tout attachement aux choses et aux êtres de ce monde et étaient donc hostiles aux passions charnelles. L'homme vertueux devait, certes, se marier pour procréer et ne rechercher que la procréation dans les embrassements conjugaux. Comment cette doctrine délibérément hostile à l'amour a-t-elle pu trouver audience chez les disciples de ce Christ qui avait mis l'amour au cœur de sa révélation ? Ce paradoxe mérite d'être souligné et expliqué [4].

\section{L'ORTHODOXIE AFFRONTE LES GNOSTIQUES}

Alexandrie fourmillait de sectes religieuses, dites gnostiques. Pour les orthodoxes, ces gens-là sont des hérétiques: l'orthodoxie est ainsi contrainte de se définir par rapport à leurs doctrines.

En matière de morale sexuelle, l'orthodoxie paraît tenir un juste milieu entre des extrémismes contradictoires : d'un côté des sectes ascétiques qui condamnent toutes formes de relations sexuelles ; de l'autre des sectes indifférentes aux péchés de la chair ou faisant même d'actes sexuels pratiqués en groupe des cérémonies religieuses. En fait, il est difficile de savoir ce que fut réellement la doctrine et le comportement de chacune de ces sectes : de ceux qui par ascétisme refusaient le mariage, leurs adversaires pensaient qu'ils tombaient fatalement dans la fornication et ils les accusaient de tous les vices ; de ceux qui faisaient de l'accouplement un rite sacral, ils disaient qu'ils refusaient la procréation. On soupçonne donc d'inquiétants amalgames et, dans le détail, on se demande souvent si telle secte était ascétique ou antinomienne. On admet que ce qui fait l'unité du gnosticisme en ce domaine c'est le refus de la procréation. Les gnostiques condamnent généralement ce monde de la chair et refusent de le perpétuer.

Mais cette définition du gnosticisme suppose définie l'orthodoxie chrétienne. Car l'opposition de la chair et de l'esprit, ce dualisme fondamental, imprègne profondément le Nouveau Testament. Nulle part il ne s'intéresse à la procréation. Chacune des sectes dites gnostiques peut donc se prétendre dans la tradition du Christ et de saint Paul davantage qu'une Eglise imprégnée de conformisme païen en matière de doctrine sexuelle.

Les Encratites, disciples de Tatien et de Jules Cassien, prennent à la lettre les mots du Christ sur les eunuques "qui le sont devenus de leur propre fait, à cause du royaume des cieux » (Mt. 19,22) et ils cherchent dans la castration un remède définitif aux atteintes de la concupiscence. En cela ils suivent saint Justin qui, pour ne pas donner prise aux calomnies des païens, conseillait aux jeunes chrétiens de se châtrer. D'ailleurs au siècle suivant le grand Origène en viendra encore à cette solution. Contre la procréation ils lisent en Mt. 24, 19 et en Luc 21, 23 : «Malheur aux femmes qui sont enceintes et à celles qui allaitent en ces jours...» [6].

Au plan de la sexualité l'orthodoxie est conduite à mettre l'accent sur le bien de procréation, malgré le silence du Nouveau Testament sur ce point.

\section{L'ATTITUDE DES CHRÉTIENS DEVANT LA CONTRACEPTION}

A l'exception de saint Jean Chrysostome, tous les Pères de l'Eglise adoptèrent cette doctrine du mariage. Clément, Ambroise, Jérôme, prennent la conduite animale comme modèle de ce qui est naturel puisque, ajoutent-ils, non souillé par le péché originel. Leur réflexion sur la nature les entraîne aussi à définir la fonction propre à chaque organe sans référence à la personne dans son entier.

L'attitude des chrétiens est curieuse puisqu'ils continuent à opposer le royaume de Dieu à ce monde de chair corrompu et transitoire. Même lorsque, à partir du IV e siècle, l'Eglise sera officiellement établie dans l'Empire romain, ni le bien de l'Etat, ni le souci de triompher des païens, ne les rendront populationnistes. Une fois passée la grande menace gnostique et avant que ne se précise la menace manichéenne (Manichéisme - Manès = Persan du Ille siècle), on voit au contraire les auteurs du IVe siècle multiplier les arguments contre la nécessité de procréer, pour défendre la prééminence de la virginité. Ambroise prêche sur Luc 23, 29 - « Heureuses les stériles... " ; Jérôme donne en exemple le mariage virginal de Joseph et de Marie. Commentant le "croissez et multipliez " de la Genèse, saint Basile refuse de l'entendre de la même façon pour l'homme et pour les animaux : l'homme n'est pas invité à se reproduire et croître en nombre comme ceux-ci, mais à croître en raison pour dominer le monde (Sermons, l, 17-18).

Examinant la différence entre l'attitude chrétienne et l'attitude juive à l'égard du mariage, Eusèbe de Césarée y discerne trois motifs: un motif eschatologique - les juifs s'intéressent aux commencements, les chrétiens à la fin ; un motif stratégique les juifs, dans un monde encore peu peuplé, devaient procréer pour transmettre la révélation tandis que les chrétiens peuvent la transmettre par la parole à des foules innombrables ; enfin les chrétiens doivent tellement se consacrer au service divin qu'ils n'ont pas le loisir d'élever une famille. Tertullien (155-220) affirmait au III ${ }^{\mathrm{e}}$ siècle dans son Apologie que la population augmente sans cesse et que peste, famine, guerres sont des remèdes nécessaires à cet accroissement. Au IV siècle saint Jérôme (347-420), lui faisant écho, s'écrie : « Le monde est déjà plein et la terre ne nous contient plus ». Enfin pour saint Jean Chrysostome (340-407), patriarche de Constantinople, aussi « le monde est rempli " [2].

Lorsque les chrétiens se réfèrent à la procréation c'est pour légitimer les rapports sexuels et donner un contenu au concept de chasteté conjugale.

Contre les autres procédés de limitation des naissances, les condamnations foisonnent d'ailleurs dès la fin du ler siècle. A cette date, un texte déjà antignostique, la Didaché, attaque les «tueurs de descendance, corrupteurs du plasma divin ». Au Ile siècle, l'Epittre de Barnabé jette l'anathème sur ceux qui imitent la belette, « cet animal qui conçoit avec sa bouche » ; saint Justin (100165), dans son Apologie, loue une femme qui a quitté son mari pour échapper aux pratiques honteuses qu'il lui imposait ; saint Clément d'Alexandrie (150-211) interdit de gaspiller ni polluer la semence ; Marcus Minucius Felix (apologiste du Ille siècle), dans son Octavius, fustige les païennes qui «en buvant des drogues, éteignent la naissance d'un homme futur et, avant que de porter, commettent un parricide ». D'autres écrits, entre 220 et 230 , réprouvent les " atokiois pharmakois ", ces drogues que le médecin grec Soranos d'Ephèse (ler siècle) décrit 
clairement comme contraceptives. II paraît, en effet, certain que les chrétiens de cette époque considéraient l'usage de ces drogues comme un meurtre [7].

Accuser la contraception d'être, comme l'avortement, destructrice d'un être humain sera en effet habituel chez les auteurs chrétiens postérieurs. Peut-être les théories anciennes de la génération sontelles responsables d'une certaine confusion entre avortement et contraception. Pour Aristote (384-322) le sperme viril est un principe actif qui organise la matière féminine - le sang menstruel - pour façonner le fœetus. Pour Soranos, le sang menstruel ne sert que d'aliment à la semence virile qui croit d'elle-même, comme une graine, une fois semée dans l'utérus.

Mais pas plus qu'Aristote, Soranos ne croit pas que cette semence soit, dès l'émission, douée d'une âme. Ni la théorie stoïcienne, ni la tradition hébraïque ne lui en accordent non plus. De sorte que, si ces théories permettaient d'assimiler l'avortement avant animation du fœtus à un acte contraceptif, elles ne permettent pas d'assimiler la contraception à un avortement. Le respect de la vie ne peut avoir été la cause profonde de l'hostilité chrétienne aux manœuvres contraceptives ; faire de la contraception un meurtre fut essentiellement un artifice rhétorique : après avoir assimilé l'infanticide à un parricide et l'avortement à un infanticide, on a fait de la contraception un avortement [5].

Saint Jean Chrysostome est aussi hostile à la contraception. Dans son Épitre aux Romains, il condamne de manière véhémente les rapports avec une prostituée parce qu'ils impliquent la contraception et que celle-ci est «pire que le meurtre». Selon Chrysostome, l'accouplement conjugal est un rite sacré ordonné par Dieu cornme témoignage d'amour. II n'entraîne pas de luimême la procréation, mais de par la puissance de Dieu. Et si la contraception est pire que le meurtre, c'est qu'elle corrompt ce rite sacré. Ces idées dans l'immédiat auront peu d'échos en Occident et ce sont celles de saint Augustin (354-430) qui vont s'y imposer.

\section{LA DOCTRINE AUGUSTINIENNE DU MARIAGE}

Saint Augustin insiste d'abord sur ce qui a constitué l'essentiel de l'institution de mariage dans la plupart des civilisations : sa raison sociale. « Dieu a voulu, écrit-il dans son De bono conjugali, tirer tous les hommes d'un seul pour qu'ils fussent maintenus en société non seulement par la ressemblance de leur race, mais encore par le lien de parenté ". Les trois biens de mariage qui sont les composantes de ce bien essentiel sont : premièrement la procréation et l'éducation chrétienne des enfants; deuxièmement le renoncement aux rapports extra-conjugaux et le dû conjugal sur le plan sexuel comme sur celui de l'assistance mutuelle ; troisièmement la stabilité, l'indissolubilité du mariage. Une fois engagé dans un mariage stérile, un époux, même s'il pouvait trouver la fertilité dans un autre mariage, ne peut rompre le premier pour en contracter un second. C'est le " sacramentum " qui rend le mariage chrétien différent des mariages juifs ou païens, solubles pour raison d'adultère ou de stérilité [1].

Ces trois biens de mariage ne sont pourtant pas autant de raisons de rechercher l'union charnelle. Nulle part Augustin ne fait intervenir le "sacramentum " comme fin justifiante de l'accouplement. D'amour, il n'est pas question, et la fidélité apparaît au contraire dans un contexte presque juridique : elle oblige l'époux qui n'en ressent pas l'envie à s'unir à celui qui la ressent. Quant à l'époux qui recherche l'accouplement, seul peut le justifier le désir de procréation. Non seulement toute manœuvre contraceptive est rigoureusement proscrite, de même que les accouplements en période de stérilité, mais on considère comme coupables les rapports conjugaux qui n'ont pas la procréation pour fin délibérée.

Pour les Pères d'Alexandrie, déjà, le péché originel était en relation étroite avec l'acte sexuel. Saint Clément croyait qu'Adam, entraîné par la concupiscence, avait prématurément usé de la chair. Origène d'Aléxandrie (183-252) tenait le péché originel pour responsable de l'appétit pour la nourriture et le plaisir sexuel. Saint Ambroise (340-397) - archevêque de Milan - partageait avec eux l'opinion que le caractère sexuel de la génération est ce qui transmet ce péché de père en fils. Plus tard, saint Athanase (340-393) - patriarche d'Aléxandrie - saint Basile (329-379) évêque de Césarée de Cappadoce -, saint Grégoire de Nysse (335-395) - évêque de Césarée -, saint Jean Chrysostome avaient abandonné cette association causale. Mais Augustin l'affirme à nouveau, en admettant que la diversité des sexes, l'union dans le mariage, la fécondité humaine viennent de Dieu et soient bonnes. Mais il y a aussi, dit-il, la concupiscence de la chair qui arrache certains de nos membres à l'empire de notre volonté. Cette force ne vient pas du Père, mais du Satan. Accusè d'emprunter ces concepts au manichéisme, il est conduit à insister davantage encore sur ce qui le sépare des manichéens : le bien de procréation. La concupiscence étant inévitablement présente dans l'acte sexuel, celui-ci n'est légitime qu'à condition d'avoir pour fin un bien, la procréation.

\section{LA LIMITATION DE LA FAMILLE DANS L'OCCIDENT CHRÉTIEN}

Cette doctrine augustinienne qui n'était qu'une part de la tradition chrétienne et pouvait être corrigée par celle de saint Jean Chrysostome ou d'autres Pères grecs, devint du $\mathrm{VI}^{\mathrm{e}}$ au $\mathrm{XIX}^{\mathrm{e}}$ siècle la doctrine de l'Eglise romaine. Les auteurs médiévaux, malgré certains gauchissements et des précisions de détail, n'écrivirent rien qui différât de ce qu'avait écrit Augustin. En effet, la doctrine augustinienne semble avoir régné sans partage, en théorie.

\section{LA LÉGISLATION ET LES MOEURS DANS L'EMPIRE CHRÉTIEN DE L'OCCIDENT}

Les lois de la Rome païenne n'avaient jamais interdit l'infanticide. En revanche, elles se sont souciés de l'avortement et de la contraception. Elles ne visaient alors pas à protéger la vie de l'enfant à naître, mais d'abord les droits du mari.

Après la conversion de Constantin le Grand (270-337) les chrétiens obtinrent l'abrogation des lois d'Auguste limitant la capacité d'héritage des célibataires et celle des couples sans enfants parce que ces lois gênaient l'essor de la chasteté dans le célibat et dans le mariage. Du point de vue démographique cette abrogation favorisait le malthusianisme. Cependant, dès 318 on limitait la toute puissance du père sur ses enfants et depuis 374 l'infanticide fut légalement tenue pour homicide. En matière d'avortement, et de contraception la législation de l'époque chrétienne paraît rester assez loin de ces sermons où l'un était qualifié de parricide et l'autre tenu pour « pire encore que le meurtre ". Quant aux pratiques contre nature, elles sont punies du bûcher lorsqu'il s'agit d'homoséxualité masculine, mais ne paraissent pas avoir été réprimées dans les autres cas, et particulièrement en mariage.

Hors des indications que peut fournir la législation, que pouvons- 
nous savoir de la pratique en ces derniers siècles de l'empire d'Occident ?

En ce qui concerne l'intention procréatrice qui aurait dû présider aux rapports conjugaux, Augustin écrit : " Jamais, dans les conversations amicales, je n'ai entendu un homme marié ou l'ayant été déclarer qu'il ne s'est uni avec sa femme que dans le but d'avoir des enfants ". Sur les rapports avec les prostituées et la contraception qu'ils impliquaient, nous avons le sermon de Chrysostome, adressé à des chrétiens. Et sur les mœurs des jeunes filles de la haute société catholique de Rome, la Lettre 22 ad Eustochium de Jérôme. II y déplore le nombre des vierges qui faillaient quotidiennement. Certaines d'entre elles se pavanaient en dissimulant une grossesse importune sous des vêtements appropriés. D'autres prenaient « à l'avance une potion de stérilité, assassinant un être humain qui n'est pas encore né ». D'autres enfin après avoir conçu, se servaient de poisons pour provoquer des avortements. Retenons donc de ce témoignage que des bruits d'inconduite, de grossesses illégitimes, d'avortements, de contraception couraient à Rome sur ces filles de la société chrétienne dont la tenue choquait.

Sur la limitation de la famille, enfin, on peut citer le texte célèbre donné par Augustin dans Mariage et concupiscence. Parlant de ceux qui recherchent le plaisir en mariage et « mettent obstacle à la génération soit en intention soit en acte ", il écrit que "les uns poussent la perversité jusqu'à exposer les enfants qu'ils ont malgré eux » tandis que d'autres vont « jusqu'à demander au poison les moyens de demeurer stériles et, s'ils ne peuvent y parvenir... jusqu'à étouffer... dans le sein même de la mère l'enfant déjà conçu " Dans ce texte sont mentionnés des cas d'avortement ou de contraception. Les uns et les autres sont-ils devenus exceptionnels ? ou restent-ils de pratique courante? Difficile à répondre vue la pauvre documentation.

\section{LE HAUT MOYEN ÂGE ET LE TÉMOIGNAGE DES PÉNITENTIELS}

Du $\mathrm{VI}^{\mathrm{e}}$ au $X \mathrm{I}^{\mathrm{e}}$ siècle, nous disposons des documents très originaux : les pénitentiels. II apparaît d'abord que l'infanticide n'a pas totalement disparu et est lourdement frappé. On peut cependant supposer qu'il se pratique beaucoup moins que dans l'antiquité païenne. L'avortement est évoqué plus souvent encore et avec plus de précision. D'une part on distingue aussi le cas de la pauvresse de celui de " la fornicatrice cherchant à celer* sa scélératesse ». D'autre part la gravité du crime dépend du degré de formation de l'embryon : avant quarante jours l'avortement n'entraîne généralement qu'un an de pénitence ou moins encore ; après l'animation, la peine est de trois ans, parfois même de quatre, sept ou dix ans. Enfin il semble que lorsqu'on avorte par le moyen de « maléfices » - c'est-à-dire de potions magiques ou d'autres sorcelleries - la faute soit plus grave que lorsqu'on emploie un moyen mécanique.

Les drogues stérilisantes sont aussi des maléfices et on les évoque généralement en compagnie des aphrodisiaques, des abortifs, des poisons et autres moyens de sorcellerie. C'est comme sorciers que leurs utilisateurs sont plus lourdement frappés que les meurtriers. De surcroît les confesseurs enquêtent sur l'utilisation volontaire, sur soi-même, de ces stérilisants.

Donc, à cette époque la contraception existe, au moins en intention.

Les époux qui cherchaient à éviter la conception par des relations contre nature - coït anal ou commerce oral - sont eux aussi frappés plus lourdement que les meurtriers : leur pénitence durait de trois ans à tout le reste de leur vie. Quant au coït interrompu - le crime d'Onan -, sur vingt pénitentiels étudiés il n'est mentionné que deux fois et la pénitence indiquée - deux à dix ans - nous renseigne mal sur son statut particulier. Le silence des dix-huit autres pénitentiels à son égard est significatif de sa rareté ? On peut en douter. Car il est clair que les actes aboutissant volontairement à la perte de la semence ne sont pas tous frappés avec la même sévérité. La masturbation, très fréquemment évoquée, n'entraîne que quelques jours à quelques semaines de pénitence. D'autre part plusieurs pénitentiels considèrent qu'un acte de fornication est plus grave lorsqu'il se révèle stérile. Bref, il est possible que l'on ait pratiqué le coït interrompu ou l'accouplement «inter femora» pour diminuer le péché dans les relations fornicatrices et éviter le scandale d'une grossesse illégitime.

Les pénitentiels sont silencieux sur les rapports pendant la grossesse que tant de Pères condamnaient, et ils ne considèrent pas explicitement comme peccamineux ${ }^{* *}$ les accouplements sans intention procréatrice.

En ce temps des moines, l'insistance sur la chasteté, la vogue du célibat, le scandale de la procréation illégitime, s'accompagnent d'une relative indulgence à l'égard de la masturbation et de certaines relations hétéro-sexuelles intrinsèquement stériles. Sans condamner le mariage en théorie, tout se passe comme si on oubliait qu'il est le remède à la fornication. Tout se passe comme si, dans la pratique, les moines s'alignaient sur ces sectes ascétiques que l'orthodoxie accusait quelques siècles plus tôt de favoriser à titre de moindre mal le sacrilège d'actes sexuels non procréatifs.

Pour l'acte contre nature, saint Thomas d'Aquin (1225-1274) écrit : «Le désordre dans l'émission de la semence concerne la vie de l'homme en puissance... L'acte contre nature, dans son essence, menace la conservation de l'espèce humaine, fait injure au Créateur"».

Il est extrêmement difficile de savoir quels actes précis entrent dans cette catégorie. On insiste généralement sur la stérilité de ces actes ; mais la stérilité ne les définit pas assez précisément puisque l'accouplement des époux stériles, ni l'union pendant la grossesse n'entrent jamais dans cette catégorie. Saint Thomas l'explique en distinguant les actes « intrinsèquement stériles " de ceux qui ne le sont que « par accident », c'est-à-dire en raison de l'âge, du moment des rapports, de la grossesse ou de la maladie des époux. Mais d'autre part si les actes sexuels intrinsèquement stériles - ceux qui n'aboutissent pas à l'intromission du semen dans le vagin - semblent être tous contre nature, d'autres, possiblement féconds, le sont aussi en raison seulement de la position des conjoints au cours de l'accouplement. II faut alors admettre que les personnes mariées ont un ministère sacré à accomplir dont le rite a été fixé par le Créateur.

Cependant, l'accouplement normal paraît si peu sacré que beaucoup de théologiens, dès le XIIIe siècle, admettent les positions anormales lorsqu'on y est contraint par l'obésité ou par une grossesse avancée, le fruit de l'épouse risquant alors d'être "gâté " par l'accouplement ordinaire. Ces positions ne sont finalement contre nature que lorsqu'elles ont pour fin de procurer un plaisir exacerbé.

Le crime contre nature, enfin, figure très souvent dans ces listes de péchés réservés à l'évêque. Mais il est difficile de préciser quels 
actes précis sont visés. D'une manière générale, il semble que le mot désigne toujours la sodomie et parfois la bestialité. II paraît que la masturbation n'est pas réservée à l'évêque ainsi que le coït interrompu.

\section{LES MÉTHODES DE CONTRACEPTION ET LEURS UTILISATEURS}

Plusieurs ouvrages du moyen âge mentionnent des produits considérés comme stérilisants. La femme « qui boit des herbes afin de ne pas concevoir » et celles qui mettent " des choses matérielles au lieu secret ", c'est-à-dire des pessaires, sont dénoncées. Si certains produits ont un effet physique ou chimique, d'autres paraissent n'avoir eu qu'une action magique.

II ne semble pas qu'entre ces divers produits les théologiens aient établi de distinctions morales. C'est d'ailleurs aux péchés contre nature qu'ils s'intéressaient surtout. On a pourtant soutenu que si les prêtres restaient vagues dans leurs dénonciations c'est que ces actes contre nature n'avaient pas d'existence réelle dans la société du temps. En fait cette discrétion peut s'expliquer par la volonté d'assimiler la gravité de ces différents actes à celle du plus anciennement condamné : la sodomie. Cette discrétion n'est pas totale et il semble que tous les auteurs aient pensé au coït interrompu aussi bien qu'à la masturbation et la sodomie. Certains se réfèrent clairement à Onan « qui répandait sa semence en terre » ; d'autres parlent de « pollution qui n'a pas lieu dans l'organe destiné à cela par la nature " mais « dans un autre organe ou à l'extérieur » [9].

En tête des utilisateurs de "poisons de stérilité ", on dénonçait ceux qui, par jalousie, par haine ou pour s'assurer un héritage, voulaient empêcher un couple d'être fertile ou de consommer son mariage. On fait ensuite allusion à la fille qui voulait cacher sa fornication ; aux clercs qui fournissaient des contraceptifs à des femmes dont ils avaient obtenu les faveurs ; aux épouses qui cherchaient à éviter la grossesse ; aux prostituées.

Parmi les excuses de la faute, on cite dès le XIIe siècle la crainte d'une grossesse que peut avoir une femme malade. Une autre circonstance atténuante c'est la pauvreté. A ces pauvres, on propose l'étreinte réservée. Cette pratique paraît avoir été admise d'abord au $X \|^{e}$ siècle non pas pour soulager la pauvreté mais pour permettre à l'homme de rendre le dû conjugal à sa femme sans pour autant tomber dans le péché véniel que comporte le plaisir accompagnant l'éjaculation.

Après ce temps des moines (XIIE et XIIIe siècles) une nouvelle étape est marquée, comme celle du lle et des IVe-Ve siècles, par la lutte contre des hérésies pessimistes. Sans remettre en cause la prééminence de la virginité ni la continence au sein même du mariage, saint Thomas soulignait en effet leur contingence : il faudrait, dit-il, les abandonner, voire revenir à la polygamie du temps des patriarches, au cas où l'humanité serait menacée de disparition. Et il donne de la procréation des justifications inconnues de l'antiquité.

Les théologiens, cependant, continuaient à refuser le populationisme à tout prix. D'ailleurs la qualité des enfants primait leur quantité : l'interdit frappant l'accouplement en période menstruelle était expliqué par le souci de leur santé physique.

Quant aux pratiques contraceptives, l'un des faits les plus remarquables du XVIe siècle est le durcissement des condamnations qui les frappaient.

A partir du XIXe siècle il est clair que l'Eglise constitue une force de résistance à l'évolution d'une civilisation qu'elle ne contrôle plus que très partiellement. Dès la première moitié du XIXe siècie, on constante un net essor du malthusianisme.

Thomas Malthus (1766-1834) est un protestant qui reprend la tradition pessimiste des Pères de l'Eglise et de certains théologiens médiévaux. Comme saint Jérôme s'effrayant de ce que " la terre ne nous contient plus ", il prêche en faveur de la continence et du célibat prolongé. Son originalité, c'est de compter parmi les remèdes à la surpopulation, à côté de cette « vertu » nécessaire, non seulement la peste, la famine et la guerre mais le "vice ", qu'il condamne d'ailleurs sans équivoque.

Malthus n'est cependant ni le premier ni le seul théoricien du malthusianisme. Un an avant qu'il ait publié son Essai sur le principe de la population (1798), Jérémie Bentham (1748-1832) avait pour sa part préconisé la contraception. Puis Francis Place (1771-1854), ouvrier autodidacte, s'en fait l'apôtre : en 1822, il publie Illustrations et preuves sur le principe de la population, et il distribue par ailleurs aux ouvriers des tracts anonymes préconisant l'usage du coït interrompu ou d'éponges occlusives [8].

En France, philosophes et « patriotes » abreuvés aux sources de la vertu antique, ont au contraire dénoncé une dénatalité précoce qui leur paraissait l'effet de l'injustice fiscale et du célibat ecclésiastique. Pourtant, ce défaut d'expression théorique n'empêche pas la France d'être dans les faits le pays pilote en matière de contraception. Dès la fin du XVIIle siècle, la pratique du coït interrompu se diffuse massivement. Les théoriciens anglais fondent d'ailleurs sur l'exemple français leur apologie de ce moyen contraceptif, et les statistiques démographiques témoignent de son efficacité.

Le mouvement malthusien à partir de 1877 trouve la faveur du public anglais grâce au procès que le gouvernement britannique a intenté aux distributeurs d'une apologie de la contraception, The fruit of Philosophy. En un an, deux cent mille exemplaires sont vendus et la ligue malthusienne, réorganisée, essaime bientôt dans les autres pays d'Europe et d'Amérique. En France la propagande malthusienne se développe chez les ouvriers dans la dernière décennie du XIXe siècle. A partir de 1900 les ligues malthusiennes sont assez nombreuses pour organiser des congrès internationaux. De nouvelles techniques contraceptives sont d'ailleurs apparues qui font vivre une industrie florissante : dès 1843 la vulcanisation du caoutchouc a permis la fabrication massive de préservatifs solides et bon marché ; et vers 1880 est mis au point le diaphragme vaginal [10].

Quelles qu'en soient les méthodes, la limitation des naissances s'accroît. Dans la plupart des pays d'Europe occidentale les taux de natalité baissent de manière significative avant la fin du XIXe siècle. La plupart des Etats s'inquiètent d'une baisse de fécondité des mariages qui n'entraîne plus seulement un vieillissement mais déjà une diminution de la population. Partout en Europe on vote des lois favorisant la natalité.

\section{REFERENCES}

1. BRUGAROLA M. : Sociologia $y$ theologia de la natalidad. Studium Edit., Madrid, 1967, 716.

2. DUTTA R. : History and development of fertility control. In : Practice of fertility control, by S.K. Chaudhuri, 1 vol., Calcutta, Current Book Edit., 1983: 11-22.

3. FLANDRIN J.L. : L'église et le contrôle des naissances. Paris, Flammarion, 1970, 139. 
4. FONTANILLE M.T. : Avortement et contraception dans la médecine gréco-romaine. Paris, Searle, 1977 : 13-20.

5. HARRIS W. : The theoretical possibility of extensive infanticide in the Graeco-Roman World. Classical Quartely, 1982, 32 : 116.

6. HIMES N.E. : Medical history of contraception. New York, Gamut Press, 2 e édit., 1963 : 23-28.

7. MCLAREN A. : Histoire de la contraception. Paris, Noêsis, 1996 : 119-160.

8. NETTER A., ROZENBAUM H. : Histoire illustrée de la contraception de l'Antiquité à nos jours. Paris, Roger Dacosta, 1985 : 427-433.

9. NOONAN J. : Contraception et mariage. Paris, Édition du Cerf, 1969, 722.

10. SUITTERS B. : The history of contraceptives. London, IPPF, 1967 : $30-42$.

Manuscrit reçu : décembre 2007 ; accepté décembre 2007.

\title{
Notes du Rédacteur en Chef
}

(Dictionnaire historique de la langue française, Alain Rey, Paris, Le Robert, 1992).

* celer: v. tr., hérité du latin celare "cacher», mot ancien et usuel appartenant à une racine indo-européenne ${ }^{\circ} \mathrm{kel}$-, représentée dans un grand nombre de mots latins (dont sont issus en français : cellule, cil, clandestin, couleur, occulte); signifie «tenir caché, dissimuler», a pâti de la concurrence de "cacher» dans l'usage courant et fonctionne de nos jours comme son doublet stylistiquement noble et archaïque.

${ }^{* *}$ peccamineux : adj., emprunt didactique (XVIIe s.) au latin médiéval peccaminus "de la nature du péché» (XVIIIe s.), luimême dérivé de peccamen, nom du péché tiré du verbe peccare ("commettre une faute, une erreur, se tromper», spécialisation à partir du sens classique de "brancher, faire un faux-pas"), est d'abord un terme de l'ancienne médecine (humeurs peccamineuses) repris tardivement (1884, Huysmans) dans la signification religieuse «de la nature du péché».

\author{
ABSTRACT \\ The part of the Church and Christian morals on fertility \\ control from the Antiquity to the present time \\ George ANDROUTSOS, Christos ZOURNAS,
Aristide DIAMANTIS \\ This article discusses the role of the Church in birth control \\ from Antiquity to the present day.
}

Key words : church, Christian morals, birth control 